\title{
Distortion of gravitational-wave packets due to their self-gravity
}

\section{Citation}

Kocsis, Bence, and Abraham Loeb. 2007. "Distortion of Gravitational-Wave Packets due to Their Self-Gravity." Physical Review D 76 (8). https://doi.org/10.1103/physrevd.76.084022.

\section{Permanent link}

http://nrs.harvard.edu/urn-3:HUL.InstRepos:41417262

\section{Terms of Use}

This article was downloaded from Harvard University's DASH repository, and is made available under the terms and conditions applicable to Other Posted Material, as set forth at http:// nrs.harvard.edu/urn-3:HUL.InstRepos:dash.current.terms-of-use\#LAA

\section{Share Your Story}

The Harvard community has made this article openly available.

Please share how this access benefits you. Submit a story.

\section{Accessibility}




\title{
Distortion of Gravitational-Wave Packets Due to their Self-Gravity
}

\author{
Bence Kocsis 1,2 , * and Abraham Loeb ${ }^{1, \text { 田 }}$ \\ ${ }^{1}$ Harvard-Smithsonian Center for Astrophysics, 60 Garden Street, Cambridge, MA 02138, USA \\ ${ }^{2}$ Institute of Physics, Eötvös University, Pázmány P. s. 1/A, 1117 Budapest, Hungary
}

(Dated: October 25, 2018)

\begin{abstract}
When a source emits a gravity-wave $(\mathrm{GW})$ pulse over a short period of time, the leading edge of the GW signal is redshifted more than the inner boundary of the pulse. The GW pulse is distorted by the gravitational effect of the self-energy residing in between these shells. We illustrate this distortion for GW pulses from the final plunge of $\mathrm{BH}$ binaries, leading to the evolution of the GW profile as a function of the radial distance from the source. The distortion depends on the total GW energy released $\epsilon$ and the duration of the emission $\tau$, scaled by the total binary mass $M$. The effect should be relevant in finite box simulations where the waveforms are extracted within a radius of $\lesssim 10^{2} M$. For characteristic emission parameters at the final plunge between binary BHs of arbitrary spins, this effect could distort the simulated GW templates for LIGO and LISA by a fraction of $10^{-3}$. Accounting for the wave distortion would significantly decrease the waveform extraction errors in numerical simulations.
\end{abstract}

PACS numbers:

\section{INTRODUCTION}

The observation of gravitational waves (GWs) is expected to open a new window on the universe within the following decade. First generation GW detectors (InLIGO [76], VIRGO [77], TAMA 78], GEO [79]) are already operating at or close to their design sensitivity levels and the development of the next advanced-sensitivity GW detectors (Advanced LIGO 80], Advanced Virgo [81], LCGT [82]) and the space-detector LISA [83] are well underway. It is now increasingly important to fully understand the precise characteristics of the GW waveforms that we expect to observe.

The most luminous GW sources are expected to be associated with mergers of $\mathrm{BH}$ binaries. The physical understanding of these sources has greatly improved by recent breakthroughs in numerical relativity [1, 2, 3]. It is now finally possible to simulate the merger of a $\mathrm{BH}$ binary, from the initial circular inspiral, through the plunge to a common surrounding horizon, to the final ringdown, as the remnant settles down to a quiescent stationary Kerr-BH. It is widely believed now that existing simulations are sufficiently precise to allow targeted searches for these waveforms in real data [4]. In fact, it has been recently shown that the errors are not even limited by the numerical precision of the simulation $\left(\sim 10^{-5}\right)$, but the GW extraction method itself entails a much larger uncertainty $\left(\sim 10^{-3}\right)[5]$. In this paper, we demonstrate that the self-gravity during the propagation of gravitational radiation in the zone of wave extraction of numerical simuations leads to the distortion of the waves, corresponding to similar magnitude modifications in typical cases.

\footnotetext{
*Electronic address: bkocsis@cfa.harvard.edu
}

†Electronic address: aloeb@cfa.harvard.edu

\section{A. Description of the effect}

Let us imagine a compact spherically-symmetric configuration of matter (representing the remnant) and a rapidly expanding sphere of massless particles (representing the radiation) carrying away some of the initial mass of the system (Fig. 11). First, let us assume Newtonian gravity and spherical symmetry. In this case, the various shells are pulled back only by the gravity of the mass interior as if it was concentrated to a point mass at the center of the sphere, and the effect of the outer enclosing shells exactly cancels out. Thus, the particles on the outermost shell are always attracted by the total mass, including the mass of the radiation, but the innermost shells experience only the gravity of the remnant. Therefore, the gravity of the radiation implies that the innermost shells of radiation will be continuously catching up to the outer boundary during their journey from the source to the observer.

Do we expect an analogous effect to exist also for gravitational waves in full general relativity? First, let us consider conventional (i.e. non-gravitational) radiation. In analogy to the Newtonian gravitational pull, relativistic test particles are slowed down by gravity: the nullgeodesics in a gravitational field experience the so-called Shapiro delay [6], decreasing the radial coordinate velocity with increasing gravity. Furthermore, according to Birkhoff's theorem, the spacetime outside a spherically symmetric distribution of energy is equivalent to the spacetime of a point-mass placed at the center of the sphere, the Schwarzschild metric, and the spacetime inside a cavity is the free-space Minkowski spacetime. More generally, the spherically symmetric expansion of collisionless radiation is a known simple exact solution of the Einstein equations, the Vaidya metric [7, 8]. This solution has exactly the same characteristics as the Newtonian example, whereby various shells react only to the mass interior to them, i.e. they move on world lines ne- 


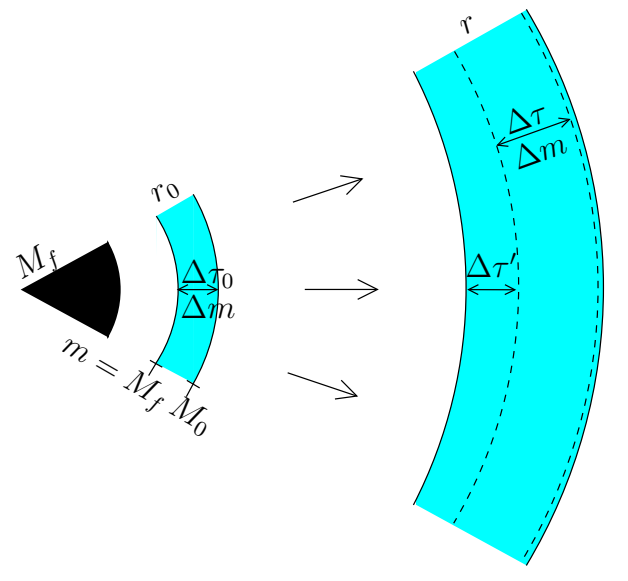

FIG. 1: A sketch of the effect under consideration. The coalescence of two BHs in a binary of total initial mass $M_{0}$ results in the emission of a burst of gravitational radiation which carries away a non-negligible $\epsilon$ fraction of $M_{0}$. The remnant $\mathrm{BH}$ mass is $M_{f}$. The proper temporal width of the wave-packet for a hypothetical observer fixed at a radial distance $r$ is $\Delta \tau$. As the packet propagates outwards, it (1) expands due to gravitational redshift of the initial mass $M_{0}$ (solid lines), (2) contracts due to the mean self-gravity of the radiation (dotted line, $\Delta \tau^{\prime}$ ), and also (3) distorts its profile due to the self-gravity of the radiation (not shown). As a result the inner shells begin to catch up, and the proper time separation in excess of gravitational redshift from the front of the burst decreases with distance. Consequently, the net luminosity of the radiation burst changes with distance.

glecting the exterior shells and the effect of the interior shells is the same as if they were concentrated to a point mass at the center.

Next, let us turn to the case of gravitational radiation. The effect of the self-energy of gravitational radiation can be accounted for in the first nonlinear-order approximation of the Einstein field equations by attaching terms of order $h^{2}$ to the stress-energy, $T_{i j}$, considering these terms as sources in addition to the regular radiation fields $[9,10,11,12,13,14,15]$. Here $h_{i j}$ is the wave amplitude which is the correction to the background metric. If the wavelength of the GW wave-packet is much smaller than the size of the wave envelope, the evolution of the wavepacket is determined by the WKB cycle-averaged effective stress-energy tensor [10, 11], independent of the specific wave-characteristics of the radiation. In this regime, we may treat the GW packet as an ensemble of relativistic particles for which our previous arguments apply. In conclusion, we anticipate that

(i) the wave-envelope will continuously expand due to the redshift of the initial mass of the binary,

(ii) it will contract due to the self-gravity of the radiation, and

(iii) in analogy to electrodynamics, we expect that the distortion of the wave envelope would lead to a continuous adiabatic modification in the GW frequency.

The purpose of this paper, is to quantify these expectations for typical $\mathrm{BH}$ merger waveforms using simple models and to demonstrate that this effect should be accounted for in relation to numerical simulations and observed merger waveforms.

\section{B. Related literature}

To our knowledge the effect of self-gravitational distortion of GWs had not been explicitly recognized previously. We elaborate on the relation of the self-distortion effect to numerical general relativity, analytical investigations like the multipolar post-Minkowskian (MPM) and post-Newtonian $(\mathrm{PN})$ theory, and the studies of the scattering of gravitational radiation in curved spacetimes.

The self-gravitational distortion of GWs is a relatively small effect on short scales currently accessible to numerical simulations. Current state-of-the-art simulations of binary $\mathrm{BH}$ mergers are restricted to the central stronggravity domain near the black holes, and extract gravity waves from the boundary of this domain. The standard method of extracting and extrapolating the waveforms to larger distances, is based on the Regge-Wheeler-ZerilliMoncrief perturbation formalism [16, 17, 18]. This is a linear-order representation of the Einstein field equations and so it neglects self-energy effects of order $h^{2}$. Cumulative nonlinear effects like the self-distortion effect should lead to systematical deformations of the linear waveform extracted at different radii, which can in principle be discovered by a rigorous convergence test. In fact, nearly all papers on simulated merger GWs study the convergence behaviour in some detail. However, due to computational limitations, the extraction radius is currently restricted to $r \lesssim 50 M$, and the extraction has been preformed on only a few, typically $3-4$ different radii with the extrapolation done empirically based on these radii $[1,2,3,4,19,20,21,22,23,24,25,26,27]$. Recently, Pazos et al. [5] reported a systematic effect of order $10^{-3}$ (for extraction radii $r \leq 80 M$ ), which is much larger than the numerical precision of the simulation $\sim 10^{-5}$. This is roughly the same order of magnitude systematic effect that we expect for the GW distortion for the given radii (see below). Note however, that Berti et al. 25] showed that the convergence behavior is also largely sensitive to simulation assumptions. On small scales probed by these simulations, other near-field nonlinearities might also be of equal importance.

A precise treatment of the waveforms at large radii is possible by analytical methods, such as the MPMexpansion introduced by Thorne [14] (see also 13]) and developed further by Blanchet \& Damour [28, 29, 30]. In Ref. 14], Thorne introduced the concept of "local-wave zone," which is outside the dynamical zone of wave generation, but where nonlinear effects are still important. In this region the propagation of gravity waves is expressed 
in terms of an expansion in powers of $h_{i j}$ as an infinite sum of multipole contributions with rapidly decreasing amplitude, which needs to be matched to the dynamical gravitational field generated by the source. The GWs in the local wave zone are given formally by the MPM expansion, whose terms correspond to different powers of the gravitational coupling constant $G$. In the PN approach, the dynamical wave generation is calculated analytically in an infinite series in the inverse speed of light $c^{-2}$. Matching the PN and MPM expansions in the local wave zone is a successful method for the calculation for steady source GWs produced by relatively slow motions, like the inspiral phase of $\mathrm{BH}$ mergers where the distance between the BHs is large enough to allow an adiabatic quasicircular orbit at $r>6 M$. To date, the PN waveforms for circular binary inspirals are available to $3.5 \mathrm{PN}$ order for general mass ratios [31] (which is the highest order that is expected to have a measurable contribution for circular inspirals by a LISA-type detector [32]) and 5.5PN order for extreme mass ratios and no BH spins 33 . To our best knownledge, the self-gravitational distortion effect has not been identified in these works. However, in this paper we show that the radius for a fixed luminosity shift or a fixed frequency shift is linearly sensitive to the energy density of the radiation. The luminosity at the inspiral phase of binaries is less than $1 \%$ of the luminosity at the final plunge 26. Therefore, even if it is negligible for inspirals, the self-gravitational modulation of GWs could be significant for the final plunge.

We expect the self-gravitational distortion of GWs to be consistent with the PN/MPM expansion, and to have corresponding PN tail counterparts [34]. The tails of GWs are caused by the scattering of linear waves on the spacetime curvature generated by the total massenergy of the source [35], which is related to the Shapiro time-delay [6] of the radiation crawling out of the background gravity of the source. At 2.5PN order beyond the Newtonian quadrupole formula, GW tails scatter off the monopole field of the remnant [36, 37, 38]. Furthermore, above $3 \mathrm{PN}$ order, the tails of the tails are produced by curvature scattering of the tails of the waves themselves, associated with the cubic nonlinear interaction between two mass monopole moments and the mass quadrupole of the source [39]. In this paper we show that the modification caused by the spherical self-gravitational distortion of GWs is to lowest order proportional to the original waveform (i.e. without this effect) times the energy density of the waveform. Since the energy density is proportional to the square of the amplitude, this possibly implies to lowest order a monopole-quadrupole ${ }^{2}$ type interaction counterpart.

The self-gravitational distortion effect is also related to the "memory effects" (or "hereditary effects") of gravitational radiation, since it is a cumulative effect that depends on the full past history of the radiation, as opposed to regular PN terms which only depend on the instantaneous retarded fields. Other known hereditary GW effects are the tails of GWs [30, 40], the Christodoulou effect [41, 42], and the GW recoil kick [43].

Finally, nonlinear effects were also examined for the interaction of plane GWs on a free space (i.e. Minkowski) background [44, 45, 46, 47, 48, 49]. The nonlinear terms in the scattering problem are found to exactly cancel up to fourth order, leaving no self-phase-modulation effect for GWs in vacuum to this order. However, the geometry of this case is very different from the one discussed in this paper where a curved background is initially present. It is also unclear whether there are self-phase modulation effects at higher nonlinear orders. Fortunately, our approach does not face such convergence issues, since we adopt the exact (i.e. non-perturbative) solution of the Einstein equations in the spherical WKB approximation of an expanding radiation shell.

The present paper aims to quantify the selfgravitational distortion effect for recently compiled merger waveforms. In $\S$ II we list the main properties of the waveforms relevant for our study. In $\S$ III we present our analysis in spherical symmetry, and derive the results for the self-gravitational distortion of signal duration and the luminosity profile. In $\S$ IV we summarize the main conclusions, and then discuss their implications. Finally, we discuss the validity of the spherical approximation and consider the possible effect of the anisotropy in the Appendix. We use units with $\mathrm{G}=\mathrm{c}=1$ and a metric signature of $(-1,1,1,1)$.

\section{MERGER WAVEFORMS}

To illustrate our effect, we adopt a simplified treatment for the merger GW waveforms. Table \lists the total radiated mass $\Delta m_{\text {tot }}$ relative to the initial total mass $M_{0}$ for various encounters between compact objects found in the literature. While all of these encounters would have a nonnegligible GW self-gravitational effect, the inspiralmerger-ringdown events are expected to have the most prominent event rates for interferometric GW detectors. Inspiral detection rate estimates are between $0.3-3 \mathrm{yr}^{-1}$ and several per day for $\mathrm{NS} / \mathrm{BH}$ mergers for inLIGO and adLIGO, respectively [50]; 3 and $100 \mathrm{yr}^{-1}$ for stellar $\mathrm{BH} / \mathrm{BH}$ inspirals in globular clusters [51] and in galactic nuclei [52] with adLIGO, respectively; $1-100 \mathrm{yr}^{-1}$ and $30 \mathrm{yr}^{-1}$ for supermassive (SMBH) and intermediate mass $\mathrm{BH}(\mathrm{IMBH})$ [53, 54, 55] and SMBH/SMBH inspirals [56, 57] with LISA, respectively. The dynamic time of the encounter is proportional to the total mass; consequently we do not consider extreme mass ratio inspiral-mergers (EMRI), as the GW luminosity of these sources is much smaller. Although high velocity stellar $\mathrm{BH}$ encounters can be very bright in GWs, these events are expected to be rare, less than $1 \mathrm{yr}^{-1}$ for adLIGO or LISA [58]. In this analysis, we focus on equal mass inspiral-mergerringdown waveforms.

Generally, the waveforms can be expanded in multi- 
TABLE I: Total radiated mass relative to the initial total mass, $\epsilon$, for bright GW encounters. For a detailed comparison of BH inspiral computations see Ref. [59].

\begin{tabular}{|c|c|c|c|c|c|}
\hline Objects & Encounter $^{a}$ & $\begin{array}{c}\text { Spins } \\
\left(\frac{S_{1}}{m_{1}^{2}}, \frac{S_{2}}{m_{2}^{2}}\right)\end{array}$ & $\begin{array}{l}\text { orien- } \\
\text { tation }^{b}\end{array}$ & Refs. & $\begin{array}{r}\epsilon \\
{[\%]}\end{array}$ \\
\hline $\mathrm{NS}-\mathrm{BH}$ & head-on & $(0.0,0.0)$ & & [60] & 0.01 \\
\hline $\mathrm{BH}-\mathrm{BH}$ & head-on & $(0.0,0.0)$ & & [61] & 0.05 \\
\hline $\mathrm{BH}-\mathrm{BH}$ & head-on & $(0.1,0.1)$ & & [61] & 0.06 \\
\hline NS-BH & orbiting & $\left(\right.$ tidal $\left.^{c}, 0\right)$ & & {$[62,63]$} & 0.1 \\
\hline $\mathrm{BH}-\mathrm{BH}$ & head-on & $(0.2,0.2)$ & & [61] & 0.12 \\
\hline $\mathrm{BH}-\mathrm{BH}$ & head-on & $(0.0,0.0)$ & & $64]$ & 0.13 \\
\hline $\mathrm{BH}-\mathrm{BH}$ & whirl & $(0.0,0.0)$ & & [24] & $0.5-3$ \\
\hline $\mathrm{BH}-\mathrm{BH}$ & grazing & $(0.9,0.7)$ & $-\perp-$ & [65] & 0.9 \\
\hline $\mathrm{BH}-\mathrm{BH}$ & grazing & $(0.9,0.7)$ & $+\perp+$ & [65] & 1.0 \\
\hline $\mathrm{BH}-\mathrm{BH}$ & grazing & $(0.0,0.0)$ & & [65] & 1.2 \\
\hline $\mathrm{BH}-\mathrm{BH}$ & inspiral & $(0.2,0.2)$ & $+\|+$ & [20] & 1.8 \\
\hline $\mathrm{BH}-\mathrm{BH}$ & inspiral & $(0.1,0.1)$ & $-\|-$ & [20] & 2.0 \\
\hline $\mathrm{BH}-\mathrm{BH}$ & inspiral & $(0.2,0.2)$ & $-\|-$ & [20] & 2.0 \\
\hline $\mathrm{BH}-\mathrm{BH}$ & inspiral & $(0.8,0.8)$ & $-\|-$ & {$[22]$} & 2.2 \\
\hline $\mathrm{BH}-\mathrm{BH}$ & inspiral & $(0.1,0.1)$ & $+\|+$ & [20] & 2.4 \\
\hline $\mathrm{BH}-\mathrm{BH}$ & inspiral & $(0.0,0.0)$ & & [20] & 2.5 \\
\hline $\mathrm{BH}-\mathrm{BH}$ & inspiral & $(0.0,0.0)$ & & {$[3,21]$} & 3.2 \\
\hline $\mathrm{BH}-\mathrm{BH}$ & inspiral & $(0.0,0.0)$ & & {$[19,22]$} & 3.5 \\
\hline $\mathrm{BH}-\mathrm{BH}$ & inspiral & $(0.0,0.0)$ & & {$[25,27]$} & $3.7^{d}$ \\
\hline $\mathrm{BH}-\mathrm{BH}$ & inspiral & $(0.1,0.1)$ & $+\|+$ & {$[26]$} & 5.2 \\
\hline $\mathrm{BH}-\mathrm{BH}$ & inspiral & $(0.8,0.8)$ & general $^{e}$ & [23] & $5-6$ \\
\hline $\mathrm{BH}-\mathrm{BH}$ & inspiral & $(0.8,0.8)$ & $+\|+$ & {$[22]$} & 6.7 \\
\hline $\mathrm{BH}-\mathrm{BH}$ & r.whirl & $(0.0,0.0)$ & & [24] & $15^{f}$ \\
\hline $\mathrm{BH}-\mathrm{BH}$ & r.head-on & $(0.0,0.0)$ & & [66] & 16 \\
\hline $\mathrm{BH}-\mathrm{BH}$ & r.head-on & $(1.0,0.0)$ & $\perp$ & [67] & $17 \frac{\eta}{1 / 4}^{g}$ \\
\hline $\mathrm{BH}-\mathrm{BH}$ & whirl & $(0.0,0.0)$ & & {$[24]$} & $24^{h}$ \\
\hline $\mathrm{BH}-\mathrm{BH}$ & r.whirl & $(0.0,0.0)$ & & [24] & $100^{h}$ \\
\hline
\end{tabular}

${ }^{a}$ Here "head-on" stands for a direct collision with $v \| r$ initially, "orbiting" stands for the tidal stripping of a NS by a BH in close orbit, "grazing" stands for inspiral collisions in which the initial separation is within the final orbit in a merger, "inspiral" is the complete inspiral-merger-ringdown event, "whirl" stands for particles approaching from infinity with some impact parameter leading to a quasi-circular whirl-type orbit before merger, "r.whirl" and "r.head-on" corresponds to relativistic initial velocities $v \approx 1$ at $r \gg M$.

${ }^{b}$ Here we give the sign of $S_{1} \cdot J$, the relationship between $S_{1}$ and $S_{2}$, and the sign of $S_{2} \cdot J$, where $J$ is the orbital angular momentum. In case of a head-on collision with spins, we give the initial direction of the spin relative to the separation vector.

${ }^{c} \mathrm{NS}$ tidally locked

${ }^{d}$ Ref [25] provides the results for different mass ratios, $m_{1} / m_{2}=$ $1-4$, and found that $\Delta m \propto \eta^{2}$, where $\eta=m_{1} m_{2} /\left(m_{1}+m_{2}\right)^{2} \leq$ $1 / 4$.

${ }^{e} 8$ different choices of spin orientations

${ }^{f}$ In case the impact parameter is small enough to end up in a merger.

${ }^{g}$ Calculated for $m_{1} \ll m_{2}$. (See $\eta$ above at ${ }^{d}$.)

${ }^{h}$ In case the impact parameters are fine tuned for the binary to approach the unstable circular orbit. poles [14]

$$
h_{\mu \nu}=\sum_{n} \frac{(1+z) \mathcal{A}_{\mu \nu}^{n}}{d_{L}} e^{i \phi_{n}}
$$

where $\phi$ is the high frequency GW phase which is related to the instantaneous frequency through $f=\mathrm{d} \phi / \mathrm{d} t ; \mathcal{A}_{\mu \nu}$ is a slowly varying envelope describing how the instantaneous amplitude changes over the waveform, the index $n$ labels the various polarizations and multipoles, and $d_{L}$ is the luminosity distance, and $z$ is the redshift. For binary inspiral merger waveforms, the $(l=2, m=2)$ multipole (i.e. quadrupole) dominates the waveform [25, 26, 27]. For the sake of simplicity, we restrict our attention to a single monochromatic wave.

During a single GW cycle, corresponding to a time interval $f^{-1}$, the envelope $A_{\mu \nu}$ can be regarded as constant. In the WKB approximation, the energy carried by the radiation can be calculated as a cycle-averaged quantity. The effective stress-energy tensor of the radiation is 11, 12

$$
T^{\mu \nu}=\frac{1}{64 \pi} \frac{A^{2}}{d_{L}^{2}} k^{\mu} k^{\nu},
$$

where $A^{2}=\mathcal{A}^{\mu \nu} \mathcal{A}_{\mu \nu}$ is the squared effective amplitude and $k_{\mu}=\phi_{, \mu}$ is the wave number.

Each infinitesimal volume of the wave can be attributed the mass-energy that it carries according to Eq. (21). In the spherically symmetric approximation, the total luminosity (or more precisely the graviton number) is [1]

$$
L=\frac{\mathrm{d} E}{\mathrm{~d} r \mathrm{~d} t}=4 \pi r^{2} T^{t r}=\frac{A^{2}}{16} k^{t} k^{r}
$$

With this equation it is possible to obtain the waveform $[A(t), f(t)]$ within radius $r$ from the luminosity function $L(t)$.

Based on the waveforms derived by numerical simulations (such as Fig. 25 in Ref. [26]), we adopt the following simple fit to the effective luminosity

$$
L(t)=L_{0} \times\left\{\begin{array}{cc}
{\left[\left(t_{1}-t\right) / t_{1}\right]^{-1.5}} & \text { if }-t_{0}<t<0 \\
1 & \text { if } 0<t<t_{1} \\
\exp \left[-\left(t-t_{1}\right) / t_{2}\right] & \text { if } t>t_{1}
\end{array}\right.
$$

where the intervals $t<0,0<t<t_{1}$ and $t_{1}>t$ correspond to the late inspiral/final orbits, the peak luminosity at the plunge, and the ringdown phases, respectively, $-t_{0}$ sets the initial time of the calculated profile, $t_{1}$ represents the characteristic timescale of the most intensive part of the radiation, $t_{2}$ sets the ringdown decay rate, and $L_{0}$ is the normalization luminosity. Numerical simulations show [26] that the characteristic frequency of the radiation rapidly increases after the inspiral and saturates at $\omega=2 \pi f \sim M_{0}^{-1} / 2$ at $t \sim 0$, where $M_{0}$ is the initial mass of the source. The characteristic number of wave cycles during the brightest phase is $N=\Delta t / f^{-1} \sim 3$ 
over a time $\Delta t=12 \pi M_{0}$. Note that we assume that the WKB method is applicable to the waveform, implying that $L(t)$ does not change greatly over a single cycle. This condition is just marginally satisfied for these waveforms.

For a simple analysis, we assume that the total luminosity crossing a sphere at infinity $L_{\infty}(t)$ is given by Eq. (4) with the following parameters: $\left(t_{0}, t_{1}, t_{2}\right)=$ $(100,10,5) M_{0}$, and use $\Delta t=12 \pi M_{0}$ in reference to average quantities below. Under the WKB approximation the luminosity (4) evolves independently of the carrier frequency $f \sim 1 /\left(4 \pi M_{0}\right)$. The $L_{0}$ normalization is set using the total radiated mass of the system as a fraction of $M_{0}$ by $\epsilon=\Delta m_{\text {tot }} / M_{0}$ for the inspiral-mergers (3) listed in Table I. For pedagogical comparison purposes we distinguish the inspiral events only based on the normalization $L_{0}$, and do not consider the variations in the shape of the waveform (e.g. $\Delta t$ ).

\section{QUANTITATIVE ESTIMATES IN SPHERICAL SYMMETRY}

To give a first quantitative estimate of the magnitude of the wave-packet distortion due to self-energy, we start by computing the propagation of unpolarized radiation packets in the spherically symmetric Vaidya spacetime. The possible effect of anisotropies is discussed in the Appendix.

The Vaidya metric [7] in radiation coordinates $(u, r, \theta, \phi)$ is

$$
\mathrm{d} s^{2}=-\left(1-\frac{2 m(u)}{r}\right) \mathrm{d} u^{2}-2 \mathrm{~d} r \mathrm{~d} u+r^{2} \mathrm{~d} \Omega,
$$

where $d \Omega=d \theta^{2}+\sin ^{2} \theta d \phi^{2}$. This metric is an exact (i.e. nonperturbative) solution of Einstein's equations in spherical symmetry in the eikonal approximation to a radial flow of unpolarized radiation. Here $u$ is the retarded time parameter which is constant along the world lines of radially outgoing radiation, and $m(u)$ describes the mass function interior to $u$. Outside the radiation (i.e. where $m(u)$ is constant in the space-time), the Vaidya metric (5) is the Schwarzschild solution in EddingtonFinkelstein coordinates [12]. For our approximate waveforms decribed in $\S$ II] $m(u)$ is $M_{0}$ constant outside, it is quickly changing within a short range $0 \leq u \leq \Delta u_{\text {tot }}$ across, and it is $M_{f}=M_{0}-\Delta m_{\text {tot }}$ inside the radiation shell. Here, $\Delta m_{\text {tot }}$ and $\Delta u_{\text {tot }}$ are set by the simulated waveforms $\oint$ I.

For the metric given by Eq. (5) it is straightforward to derive the convergence of null-geodesics describing the world lines of radiation shells using Raychaudhuri's equation or the equation of geodesic deviation [68]. In either way, we find that radially outgoing shells of radiation simply follow the world lines $u(r)=$ constant, implying that the $\Delta u$ coordinate difference between the shells does not change during the propagation. The physical contraction of radiation shells can be examined using the proper time measure between shells and the observed luminosity profile.

\section{A. Proper time duration}

First, we estimate the proper-time duration of the GW signal along the world-line of a hypothetical observer crossing the radiation shell. For simplicity, we restrict to observers at a fixed spacial coordinate $(r, \theta, \phi)$. Then we have $\mathrm{d} r \equiv \mathrm{d} \theta \equiv \mathrm{d} \Omega \equiv 0$ and Eq. (5) gives

$$
\mathrm{d} \tau^{2}=-\mathrm{d} s^{2}=\left(1-\frac{2 m(u)}{r}\right) \mathrm{d} u^{2}
$$

leading to $\mathrm{d} \tau=\sqrt{1-2 m(u) / r} \mathrm{~d} u$. Therefore, $\mathrm{d} u$ can be interpreted as the infinitesimal proper time difference between two radiation shells at fixed radius approaching infinity. Thus, we adopt the notation $\mathrm{d} u \equiv \mathrm{d} \tau_{\infty}$, and similarly $\Delta u \equiv \Delta \tau_{\infty}$ for integrated quantities. Finally, let us define $m_{i}=m\left(u_{i}\right)$ for $i \in\{1,2\}$ and $\Delta m=\mid m_{2}-$ $m_{1} \mid$. We set $\left(m_{1}, m_{2}, \Delta m_{\text {tot }}\right)=\left(M_{0}, M_{f}, \epsilon M_{0}\right)$ when referring to the total GW signal duration.

Integrating between two arbitrary shells of radiation $u_{2}$ and $u_{1}$ gives

$$
\Delta \tau=\int_{\tau_{1}}^{\tau_{2}} \mathrm{~d} \tau=\int_{u_{1}}^{u_{2}}\left(1-\frac{2 m(u)}{r}\right)^{1 / 2} \mathrm{~d} u .
$$

After expanding $m(u)$ in a Taylor-series, the integrand becomes

$$
\left(1-\frac{2 m_{1}}{r}-\frac{2 u\left\langle m^{\prime}\right\rangle}{r}-\frac{u^{2}\left\langle m^{\prime \prime}\right\rangle}{r}+\ldots\right)^{1 / 2} .
$$

Substituting in Eq. (7), and using $\left\langle m^{\prime}\right\rangle=-\Delta m / \Delta \tau_{\infty}$ and $\left\langle m^{\prime \prime}\right\rangle=0$ to first order, we get

$$
\Delta \tau\left(m_{1}, m_{2}, r\right)=\left.\frac{r \Delta \tau_{\infty}}{3\left|m_{2}-m_{1}\right|}\left(1-\frac{2 m}{r}\right)^{3 / 2}\right|_{m_{1}} ^{m_{2}}
$$

Setting $m_{1}=M_{0}$ and expanding in terms of $\Delta m$, we get

$$
\frac{\Delta \tau}{\Delta \tau_{\infty}}=\sqrt{1-\frac{2 M_{0}}{r}}\left(1+\frac{1}{2} \frac{\Delta m}{r-2 M_{0}}+\mathcal{O}\left(\Delta m^{2}\right)\right) \text {. }
$$

The leading order term can be indentified as the gravitational redshift for constant mass $M_{0}$, the second term describes the correction due to the radiation mass. If we expand also in terms of powers of $1 / r$, the relative change in the proper time duration of the signal becomes

$$
\frac{\Delta \tau-\Delta \tau_{\infty}}{\Delta \tau_{\infty}}=-\left(M_{0}-\frac{\Delta m}{2}\right) \frac{1}{r}+\mathcal{O}\left(r^{-2}, \Delta m^{2}\right) .
$$

Equations (9 11) describe the self-gravitational distortion between radiation shells in terms of proper time along world lines of $r=$ const. One can notice that to 
leading order this is simply the gravitational redshift for the average enclosed mass between the shells. However, since $\Delta m$ changes along the wave packet non-trivally for a fixed radius as a function of time, the modification of the profile is generally not self-similar, leading to the distortion of the luminosity profile as a function of radius.

To make this point clearer, we correct for the average distortion of the signal and calculate the residual distortion of the signal. Let us define

$$
\Delta \tau^{\prime}=(1+z) \Delta \tau-\Delta \tau_{\infty}
$$

where $(1+z)$ is a time-independent constant representing the "average gravitational redshift" at a given $r$, given by

$$
1+z=\frac{1}{\sqrt{1-\frac{2\langle m\rangle}{r}}}
$$

$\langle m\rangle \equiv\left(m_{1}+m_{2}\right) / 2$ is the average mass, and to leading order

$$
z \approx \frac{\langle m\rangle}{r}=\frac{M_{0}}{r}-\frac{\Delta m}{2 r} .
$$

Now let us take an arbitrary radiation shell enclosing mass $\Delta m$ relative to the shell enclosing mass $M_{0}-$ $0.5 \Delta m_{\text {tot }}$, i.e. we set $m_{1}=M_{0}-0.5 \Delta m_{\text {tot }}$ and $m_{2}=$ $m_{1}-\Delta m$ in eq. (9). After correcting for the average gravitational redshift using Eqs. (12) and (13), the residual relative distortion to leading order is

$$
\frac{\Delta \tau^{\prime}}{\Delta \tau_{\infty}}=-\frac{\Delta m}{2 r}+\mathcal{O}\left(r^{-2}, \Delta m^{2}\right)
$$

Figure 2 shows the residual distortion using the exact formula (Eq. (12), thick lines) and the leading order contribution (given by Eq. (15), dotted lines). At the typical radius used by numerical simulations for waveform extraction, $r=50 \mathrm{M}_{\odot}$, the primary bulk waveform distortion changes the signal duration by $M_{0} / r \sim 2 \%$, and the secondary relative waveform distorsion between the front and the back of the signal is $\epsilon M_{0} /(2 r) \sim 7 \times 10^{-4}$ for a typical $\mathrm{BH}$ inspiral-merger with high spins $\epsilon=7 \%$ (see $\S$ III). The figure also shows that the higher order effects beyond $1 / r$ lead to an uncertainty of order $10^{-3}-10^{-4}$ for $r=(30-50) M_{0}$.

\section{B. Luminosity Profile}

Since Eqs. (9) and (11) are applicable to two arbitrary shells of radiation, we can use them to compute the evolution of an arbitrary initial radiation profile, whereas the luminosity is simply $L=\Delta m / \Delta \tau$, the total mass-energy crossing a sphere at radius $r$ within proper time $\Delta \tau$.

The profile at infinity is given by $m(u)$ in radiation coordinates, or $\Delta \tau_{\infty}(m)$, the proper time a shell enclosing mass $m$ arrives at $r=R$ where $R \rightarrow \infty$, relative to the outermost shell of radiation [84]. Here $\Delta \tau_{\infty}(m)$ can

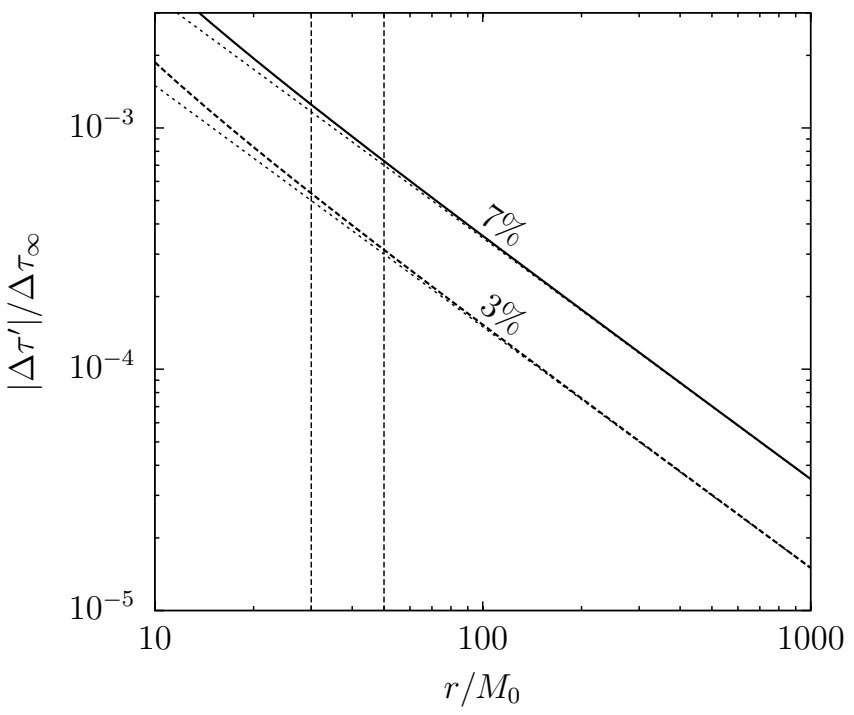

FIG. 2: The residual self-gravitational distortion of shells after correcting for the bulk gravitational redshift. The thick curves show the change in the proper time duration of the signal at radial distance $r$ from the source between shells enclosing $7 \%$ (top) or $3 \%$ (bottom) of the total mass, dotted lines correspond to the leading order term given by Eq. (15). The vertical lines highlight the typical radii used in numerical simulations for GW extraction.

be any monotonically decreasing function, for which the luminosity at $R$ in Eq. (3) is

$$
L_{\infty}(\tau)=-\left[\frac{\mathrm{d} \Delta \tau_{\infty}(m)}{\mathrm{d} m}\right]^{-1},
$$

where the minus sign originates from our definition of $m$ : the shell labeled by the largest value of $m$ arrives the earliest. The luminosity profile can also be obtained as the function of time, $L_{\infty}(\tau)$, using the relationship $\Delta \tau(m)$. Conversely, for given $L_{\infty}(\tau)$, we can compute $\Delta \tau_{\infty}(m)$ using Eq. (16). The luminosity at some other distance $r$ can be obtained similarly if given $\Delta \tau(m, r)$, the arrival time of mass $m$ relative to the outermost shell at distance $r$. This function is given by Eq. (9), substituting the waveform $\Delta \tau_{\infty}(m)$ for $\Delta \tau_{\infty}$, and $\left(m_{1}, m_{2}\right)=\left(M_{0}, m\right)$. The luminosity profile at $r$ is then

$$
L_{r}(m)=-\left[\frac{\partial \Delta \tau(m, r)}{\partial m}\right]^{-1}=\left(1-\frac{2 m}{r}\right)^{-1 / 2} L_{\infty}(m)
$$

Therefore, the modification of the profile in Bondi radiative coordinates $(m, r)$ the profile is distorted selfsimilarly. However, in terms of the observer proper time variable, $\Delta \tau(m)=\int_{0}^{m} L_{r}(m)^{-1} \mathrm{~d} m, L_{r}(\tau)$, the modification to the profile will not be self-similar:

$$
L_{r}(\tau)=\left(1-\frac{2 \int_{0}^{\tau} L_{r}\left(\tau^{\prime}\right) \mathrm{d} \tau^{\prime}}{r}\right)^{-1 / 2} L_{\infty}\left(\int_{0}^{\tau} L_{r}\left(\tau^{\prime}\right) \mathrm{d} \tau^{\prime}\right)
$$


Equation (18) relates the luminosity profile as a function of proper time at radius $r, L_{r}(\tau)$, to the profile at infinity, $L_{\infty}(\tau)$. Comparing Eqs. (17) and (18) shows the advantage of Bondi type radiative coordiantes as opposed to proper time.
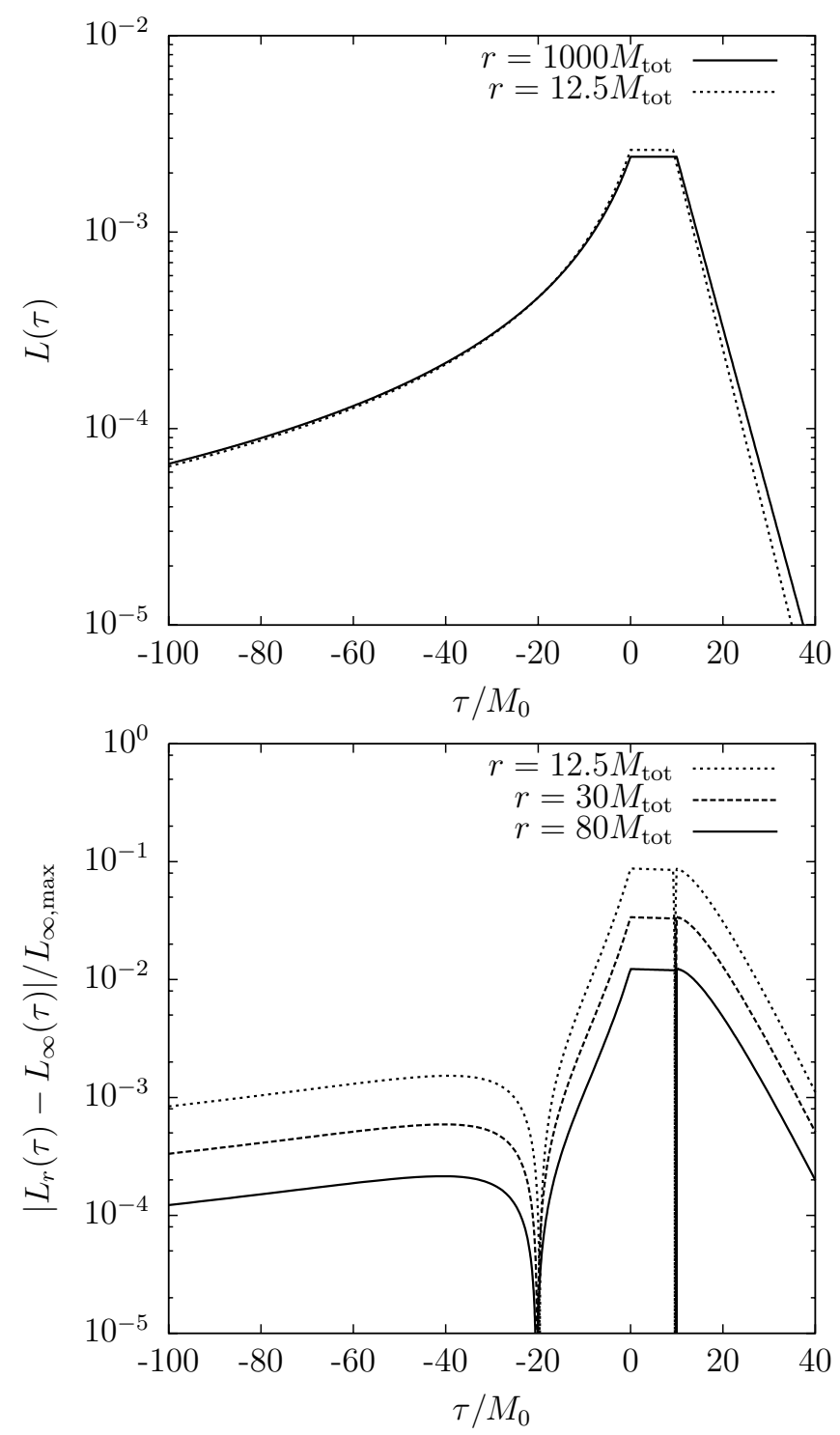

FIG. 3: Our fits to the GW luminosity profile for binary BH inspiral merger simulations as a function of observer proper time $\tau$ and the evolution of the profile at various distances, $r$. The profile parameters are given in $\S \amalg$ and $\epsilon=7 \%$. Top: The absolute profile (in units of $\mathrm{c}^{5} / \mathrm{G}$ ) is shown for two extremes, a nearby distance $\left(r=12.5 M_{0}\right)$ and far-away distance $(r=$ $\left.10^{3} M_{0}\right)$. Bottom: The difference between the GW luminosity profiles at infinity (i.e. $r=10^{3} M_{0}$ ) and three cases of smaller $r$, in units of peak luminosity at infinity. The peaks of the profiles are set to $\tau=0$. The main effect responsible for the differences seen in this figure is the bulk gravitational redshift.

Figure 3 plots $L_{r}(\tau)$ for our fit to the luminosity profile at infinity of merging binary BHs $L_{\infty}(\tau)$ with $\epsilon=7 \%$ (see $\S$ II). The top panel shows the absolute profile while the bottom panel shows the difference between the profile at some radius $r$ and the profile at infinity, such that the peak of the profiles are at $\tau=0$. The bottom panel is useful to visualize the characteristic evolution of the profile.
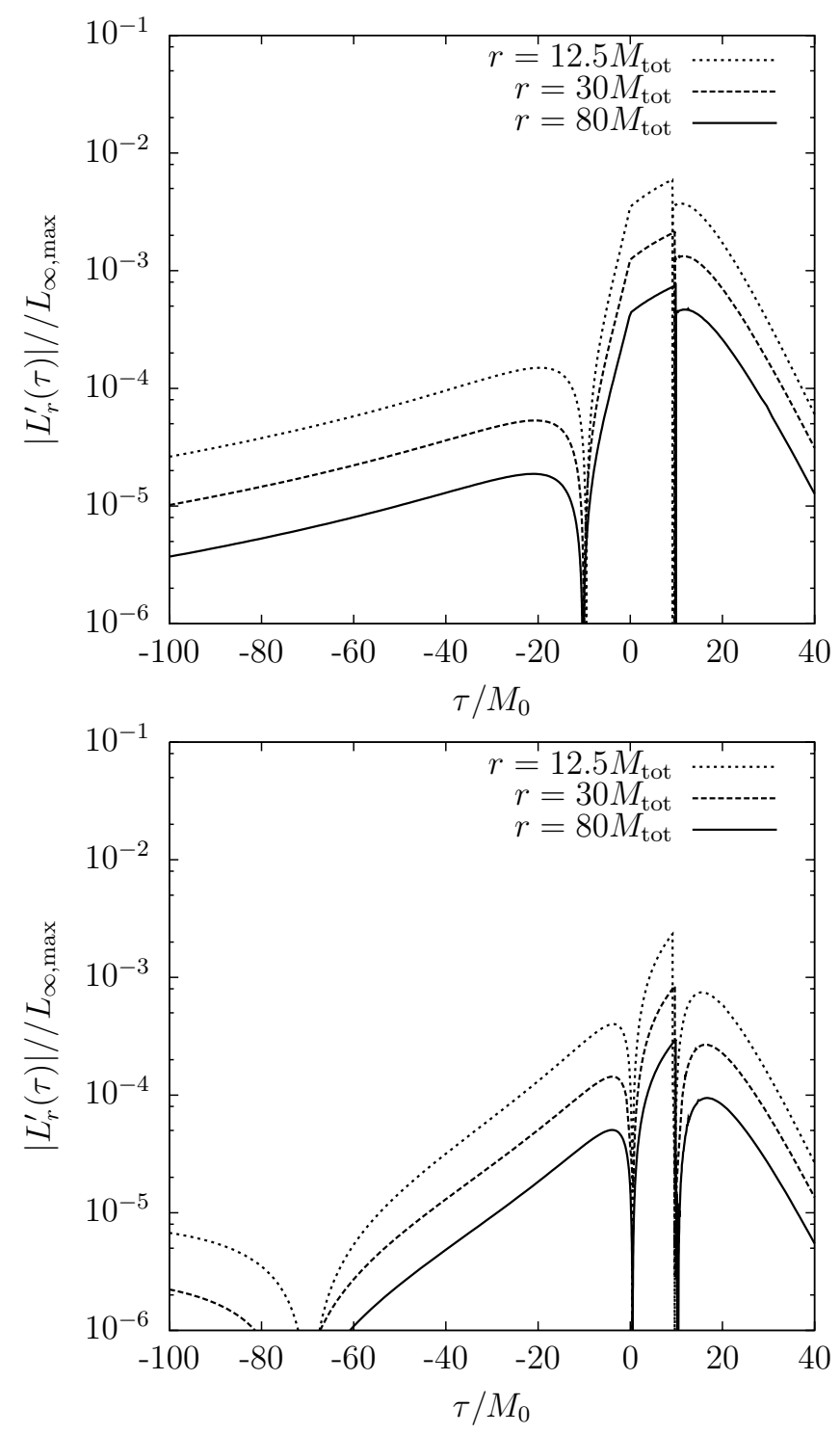

FIG. 4: The residual self-gravitational distortion to the luminosity profiles after accounting for the avarage gravitational redshift, $z=M_{0} / r$ (top) or $\left(M_{0}-0.5 \Delta m_{\text {tot }}\right) / r$ (bottom), respectively. Other parameters are the same as in Fig. 3.

In $\S$ 【II we have identified the two main effects responsible for the convergence rate of the waveform to be the gravitational redshift corresponding to the average mass and the self-gravitational effect. Indeed, the differences visible in Figure 3 are primarily due to the former. However, correcting for only the average gravitational redshift at each radius $r$ leaves a nonnegligible systematic error with respect to the true signal. To see this, we substitute $\Delta \tau_{\infty} /(1+z)$ given by Eq. (13) into Eqs. (16) and (17), and refer to the corresponding lumi- 
nosity as the average gravitational redshifted luminosity profile, $L_{r}^{z}(\tau)$. After subtracting from the true profile for each $\tau$, the residual luminosity distortion is

$$
L_{r}^{\prime}(\tau)=L_{r}(\tau)-L_{r}^{z}(\tau)
$$

where we set the reference time again to $\tau=0$ for the peak of the luminosity profiles.

Figure 4 shows the residual self-gravitational distortion $L_{r}^{\prime}(\tau)$ for various radii in units of the peak luminosity at infinity, $L_{\infty, \max }$. Naturally, the definition of the "average gravitational redshift," $z$, used for defining $L_{r}^{\prime}(\tau)$ makes a difference in the result. The top panel uses only the initial binary mass $\langle m\rangle=M_{0} / r$ in Eq. (14) totally neglecting the gravity of the radiation shell, while the bottom panel has $\langle m\rangle=\left(M_{0}-0.5 \Delta m_{\text {tot }}\right) / r$, i.e. the redshift is chosen to account also for the average gravity of the radiation shell. In the later case we find a much quicker convergence for the waveform peak at increasing radii, but the former choice is more suitable for the early parts of the waveform corresponding to the late inspiral waveform. A comparison of Fig. 3 and 4 shows that the self-gravitational distortion is roughly an order of magnitude smaller than the effect of the average gravitational redshift.

\section{Self-Gravitational Coordinate Effects}

In the previous sections we have derived the time duration and the luminosity profile of the radiation shell as it propagates radially outward from the source. We have assumed that the GW profile at each fixed arial radius $r$ is parameterized by the proper time $\tau$ of a hypothetical observer fixed at that radius, in particular the luminosity $L_{r}(\tau)$ was the total mass-energy crossing a sphere at radius $r$ within infinitesimal proper time $\mathrm{d} \tau$. Therefore, the adopted time-coordinate $\tau$ corresponds to a synchronous gauge at each radius. Since physical observables depend precisely on proper measures, these coordinates allow a simple interpretation of the convergence characteristics of the GW profile at large radii.

Other choices of coordinates would have introduced additional artificial distortion effects making the convergence characteristics of the waveforms much different. Consider for instance the "natural" coordinate system $(t, r, \theta, \phi)$ in the spherically symmetric case that is chosen to be Schwarzschild both before and after the GW burst has arrived with masses $M_{0}$ and $M_{f}=M_{0}-\Delta m_{\text {tot }}$, respectively, and which changes smoothly in between these regions. An example of such a coordinate system can be derived from the Vaidya metric Eq. (5) with the implicit transformation $t \equiv u+r+2 m(u) \ln [r-2 m(u)]$ where $m(u)$ describes the mass function interior to $u$ (which is constant along the outgoing radiation world lines). Indeed, everywhere in the spacetime where $\mathrm{d} m / \mathrm{d} u=0$, these coordinates yield a Schwarzschild metric, and the Vaidya metric in radiation coordinates (5) is then simply the Schwarzschild solution in Eddington-Finkelstein coordinates [12] in these regions. This map covers all relevant parts of the spacetime including the GW zone. The world-lines of radiation shells can be shown to follow

$$
\frac{\mathrm{d} r}{\mathrm{~d} t}=1-\frac{2 m_{u}}{r},
$$

where the second term is called the Shapiro-time delay [6] for a particle crawling out of the gravitational potential of mass $m_{u}$ interior to it. After integration, we find that to first order the temporal separation of two radiation shells enclosing mass $\Delta m$ at radius $r$ evolves to leading order as $\Delta t(r)=\Delta t\left(R_{0}\right)-2 \Delta m \ln \left(r / R_{0}\right)$, where $R_{0}$ is an arbitrary initial radius. In these coordinates, the signal duration contracts uniformly in exponential distance intervals. Even though the metric is asymptotically Minkowski (where $t$ approaches $\tau$ for $r \gg M_{0}$ ), the resulting profile evolution is fundamentally different from $\Delta \tau(r)$ given by Eq. (11)!

The appearance of the logarithmic radial dependence of the waveform was first realized by Fock [69]. This effect is specific to the harmonic coordinates and can be avoided if changing to Bondi type radiative coordinates [70, 71]. Blanchet \& Schäfer [38] have shown that a similar logarithmic dependence of the GW tail leads to a tail-induced amplitude and phase shift (typically of order $10^{-7}$ ) for stationary sources. In contrast, the logarithmic radial dependence of the wave contraction for merger waveforms can be significant for GW merger simulations. Between $r=(20-40) M_{0}$, the waveform contracts in $\Delta t$ by a fraction of $5 \times 10^{-3}$, which is just of the order of the current wave extraction precision [5, 26, 27]. This apparent logarithmic contraction effects can be avoided if one changes to the proper time variable as we have done in the previous sections. The remaining $\Delta \tau(r)$ evolution is however a physical effect.

Both the logarithmic $\Delta t(r)$ contraction and the physical $\Delta \tau(r)$ evolution (in particular the contribution denoted by $\Delta \tau^{\prime}(r)$ above) are consequences of higher order radiation effects in the Einstein equations beyond the scope of first order methods such as the Regge-WheelerZerilli-Moncrief perturbation method used for extrapolating the numerical waveforms to infinity. Therefore these effects cause the extrapolated waveforms to be different when extracting GWs from numerical simulations at various radii by standard methods using no selfgravitational interaction. For a related recent analysis see Ref. [72].

\section{DISCUSSION}

\section{A. Summary}

We considered the self-gravitational effect of gravitational radiation on the propagation of GWs from a compact source. We adopted simple approximations for the geometry of the radiation, by considering spherical symmetry on scales comparable to the radial width of 
the radiation packet. This approximation appears adequate for the quadrupolar $(l=2, m=2)$ radiation pattern around binary BH sources in numerical simulations [25, 26, 27]. Nevertheless, we use the Appendix to examine the maximal effects of anisotropy in the opposite (exaggerated) extreme, when the outgoing radiation is concentrated into a compact region. We find that irrespective of the level of anisotropy, the gravitational radiation is distorted under the influence of its own gravity as it propagates. Contrary to the standard gravitational redshift, which is a uniform shift of the waveform, the self-gravitational effect depends on the intensity and is predominant only for the most intensive bursts of radiation causing a non-uniform distortion of the waveform. The self-gravitational distortion depends on distance to leading order as $\Delta m / r$, and is therefore relevant on scales $r_{\mathrm{sg}} / M_{0} \lesssim \epsilon / \delta$ where $\epsilon$ is the radiation efficiency and $\delta$ is the desired calculation accuracy. For BH binary mergers simulations $\epsilon \sim 7 \%$ and $\delta \sim 10^{-5}$ implying that $r_{\mathrm{sg}} \lesssim 7 \times 10^{3} M_{0}$. If the GWs are extracted within this region, the self-gravitational distortion should be taken into account.

\section{B. Testing the Effect with Numerical Simulations}

Numerical simulations based on the full set of Einstein equations for binary BH inspirals have not yet reported evidence for the waveform distortion effect considered here although they have shown that the waveforms do not converge within a fractional accuracy of $\delta \sim 10^{-3}$ [5, 26, 27]. This is because the simulations are restricted to a limited volume, typically of radii $\sim(80-850) M$, while the extracted waveforms are typically compared between $r \sim 20-50 M$. For a radiation mass $\Delta m / M_{0} \sim 7 \%$, the primary effect is a shift of the waveform due to a logarithmic Shapiro time delay of the remnant, a uniform gravitational redshift, and the self-gravitational effect. We have shown that the logarithmic Shapiro delay does not show up if using proper measures to describe the waveform, and the uniform gravitational redshift is accounted for in the linear wave propagation models. However, the residual self-gravitational effect in the GW luminosity has a characteristic profile that has to be subtracted when extrapolating the extracted waveform. The peak of the effective luminosity distortion reaches $2 \times 10^{-3}$ and $5 \times 10^{-4}$ at $r=30$ and $50 M_{0}$, respectively.

Present-day numerical relativity simulations should already be capable of directly measuring the relevance of our effect by artificially amplifying the gravitational radiation found at the extraction radius $r \sim 20 M$, and starting the simulation with these amplified initial conditions. For example, for a total GW energy $\Delta m / M_{0}=30 \%$, the effective luminosity distortion between $r=20 M$ between $20-50 M$ is several percent, which is well within simulation and extraction errors. For consistency, the simulation should confirm that the total energy content of the radiation does not change. We also expect the initial ringdown frequency (corresponding to the most energetic shell) to be smaller than the final ringdown frequency.

In order to avoid errors caused by the self-gravitational distortion effect up to the desired numerical precision $\delta \sim$ $10^{-5}$, the waveform extraction radius should be chosen to be $r_{\mathrm{sg}} \gtrsim 7 \times 10^{3} M_{0}$. Alternatively, if the waveforms are extracted at smaller radii, the waveforms should be converted to Bondi type radiative coordinates and then extrapolated with the scaling $1 / r$.

\section{Observational Implications}

The self-gravitational waveform distortion is important for future observations of BH binary mergers.

1. The waveform distortion is expected to be resolvable for the LISA instrument with respect to simulated waveforms for total $\mathrm{BH}$ masses of $\left(10^{4}\right.$ $\left.10^{9}\right) \mathrm{M}_{\odot}$. The total signal to noise ratio of merger waveforms is $10^{4}$ for LISA observing $z_{c} \sim 1$ [4]. The distortion effect modifies the waveform amplitude and frequency by $\sim\left(10^{-3}-10^{-4}\right)$ for numerical waveforms extracted between $r=(30-80) M_{0}$.

2. The distortion involves a systematic modification of the waveform which needs to be accounted for in order to interpret observed merger waveforms and improve the estimation uncertainty of physical parameters beyond the uncertainty of the preceding inspiral signal. The signal to noise ratio of the final $\mathrm{BH}$ merger waveform is an order of magnitude larger than for the inspiral, implying that the merger waveform has a potential to greatly reduce parameter estimation errors. Note that the relative accuracy using only the inspiral signal with LISA is expected to be $10^{-3}-10^{-5}[73,74]$ for estimating the component masses, which is smaller than the distortion effect.

3. This effect is different from the uncertainties caused by gravitational lensing 75 , in that it is only an issue concerning the convergence properties of numerical simulations. Lensing causes an error of several percent on the inferred luminosity distance (due to the unresolved matter along the line of sight), and lensing errors increase with the source distance. In contrast, the self-gravitational effect is of order 0.1-0.01 percent for numerical simulations if the waveforms are extracted at $30 M-80 M$ and dies off quickly as $1 / r$. The wave distortion effect is of order $10^{-20}$ relative to the waveform amplitude for typical astrophysical scales. Therefore, the wave-contraction effect does not provide any additional physical parameters for observations. 


\section{Acknowledgments}

We thank George Rybicky, Irwin Shapiro and Kip Thorne for enlightening discussions. BK acknowledges support from a Smithsonian Astrophysical Observatory Predoctoral Fellowship and from NKTH Öveges József Fellowship.

\section{APPENDIX A: ANISOTROPY}

Our analysis considered only perfectly sphericallysymmetric configurations. The approach was motivated by the quasi-spherical radiation patterns found around binary $\mathrm{BH}$ sources in numerical simulations $25,26,27$. In this Appendix, we would like to examine the sensitivity of our basic results to deviations from sphericity. To gauge whether there is any such sensitivity, we analyse the most extreme case in which the outgoing radiation is concentrated into a highly compact region.

But first let us define more precisely what we assumed so far. The derivation presented in $\S$ Щrequires that the radiation field is "initially locally spherically symmetric", so that it is initially described by the Schwarzschild metric locally within some narrow solid angle $\Delta \theta \lesssim \Delta r / r$ before the GW arrives, where $\Delta r \sim c \Delta t$ is the radial width of the wave-packet along its propagation direction. But since the radiation propagating through this solid angle is in no causal contact with the radiation field expanding towards other directions, it cannot distinguish the actual spacetime from a spherically symmetric one. Note that the outermost shells of radiation expanding along different directions are always causally disconnected by definition, and the interior shells of radiation can only be affected by the outer shells within $\Delta \theta$. Since we examine the distortion effect on large distances compared to the width of the burst $r \gg \Delta r$, spherical symmetry must only be required within a very narrow angle. This simple set of considerations implies that if high-order multipoles have a vanishing contribution at large distances, the results derived in $\S$ III are applicable very generally for short bursts of radiation, $\Delta r \ll r$. Indeed, numerical simulations confirmed that the dominant contribution to the wave amplitude is given by the $(l=2, m=2)$ multipole and higher order terms are suppressed by more than a factor of magnitude (see references in $\S$ III). In the remainder of this Appendix we demonstrate the validity of this simple conclusion through explicit calculations.

We consider three variations on a toy model to estimate the effect of anisotropy. We start with the simplest model and refine this model by adding more details and complexity in the successive models. In each case, we discuss general implications for the model under consideration. In all models we consider the extreme opposite regime to spherical symmetry, namely that the radiation is maximally clumped into two outgoing BHs $L$ (leading) and $T$ (trailing) of masses $m_{L}$ and $m_{T}$, representing the leading and trailing edges of the radiation, respectively. We as- sume that $L$ and $T$ are moving in the same direction on light-like world lines, so that $T$ lies in the causal past of $L$, but $L$ is outside the causal past of $T$ throughout their propagation. We assume that there is also a remnant Schwarzschild BH $R$ with mass $m_{R}$. The instantaneous radial position coordinate of $R, T$, and $L$ at time $t$ are $0, r_{T}(t)$, and $r_{L}(t)$. We are interested in obtaining the world lines of BHs $T$ and $L$ to see how the coordinate separation $\Delta r(t)=r_{L}(t)-r_{T}(t)$ decreases with time as compared to the spherically symmetric result. In our first model we neglect the remnant $R$ (setting $m_{R}=0$ ), assume that $L$ moves with constant velocity $v_{L}$ in free space, and calculate the trajectory of $T$ in the spacetime created by $L$. Subsequently, we will generalize $L$ to move on a more general world line with a slowly changing velocity $v_{L}(t)$. Finally, we can turn on the remnant $R$ in addition to $L$, and include the retardation effect when calculating the relative motion of $T$.

We note that the spacetime of BHs moving at the speed of light have been calculated previously in Ref. [46], which found that BHs moving in the same direction do not interact. However, Ref. [46] assumed that the BHs move in free space and consequently adopted $v=1$ for their velocity. In contrast, the BHs $T$ and $L$ travel on null-geodesics in the perturbed spacetime which is initially the Schwarzschild spacetime. This difference gives rise to a non-trivial interaction between the $\mathrm{BHs} T$ and $L$.

We compare our results to the spherical case, using the $(t, r)$ coordinate system defined by Scwarzschild coordinates before and after the radiation shells as described in $\S \amalg \mathrm{IIC}$.

\section{No remnant $m_{R}=0$, constant $v_{L}$ velocity}

We start by assuming that $L$ is a $\mathrm{BH}$ with constant velocity $v_{L}<1$ in free space, and wish to calculate the world line of $T$ in this background. Here, we assume that no remnant is present, and that $L$ and $T$ move along the same spatial direction, which we denote by $x$. Thus it is sufficient to restrict our attention to the two dimensions $(t, x)$ of the spacetime.

Let us start by deriving the metric. In the coordinate system $\left(t^{\prime}, x^{\prime}\right)$ comoving with $L$, the metric is the Schwarzschild metric $\mathrm{d} s^{2}=-\left(1-\phi^{\prime}\right) \mathrm{d} t^{\prime 2}+\left(1-\phi^{\prime}\right)^{-1} \mathrm{~d} x^{\prime 2}$, where $\phi^{\prime}=2 m_{L}^{\prime} /\left|x^{\prime}\right|$. Here $x^{\prime} \equiv 0$ corresponds to the $\mathrm{BH}$ $L$ for all $t^{\prime}$, and $m_{L}^{\prime}=m_{L} / \gamma$ is the rest mass of $L$, where $m_{L}$ is the energy carried by $L$ in the original $(t, x)$ coordinates and $\gamma=1 / \sqrt{1-v_{L}^{2}}$ is the Lorentz factor. To derive the metric in the $(t, x)$ coordinate system, we apply the diffeomorphism $(t, x)=\gamma\left(t^{\prime}+v_{L} x^{\prime}, v_{L} t^{\prime}+x^{\prime}\right)$, i.e. a global Lorentz transformation,

$$
\begin{aligned}
\mathrm{d} s^{2}= & -\frac{\left(1-\phi^{\prime}\right)^{2}-v_{L}^{2}}{\left(1-v_{L}^{2}\right)\left(1-\phi^{\prime}\right)} \mathrm{d} t^{2}+\frac{1-v_{L}^{2}\left(1-\phi^{\prime}\right)^{2}}{\left(1-v_{L}^{2}\right)\left(1-\phi^{\prime}\right)} \mathrm{d} x^{2} \\
& -\frac{2 v_{L} \phi^{\prime}\left(2-\phi^{\prime}\right)}{\left(1-v_{L}^{2}\right)\left(1-\phi^{\prime}\right)} \mathrm{d} t \mathrm{~d} x
\end{aligned}
$$


which can be rearranged as

$$
\begin{aligned}
\mathrm{d} s^{2}= & \frac{\left[v_{L}+\left(1-\phi^{\prime}\right)\right] \mathrm{d} t-\left[1+v_{L}\left(1-\phi^{\prime}\right)\right] \mathrm{d} x}{\sqrt{\left(1-v_{L}^{2}\right)\left(1-\phi^{\prime}\right)}} \\
& \times \frac{\left[v_{L}-\left(1-\phi^{\prime}\right)\right] \mathrm{d} t-\left[1-v_{L}\left(1-\phi^{\prime}\right)\right] \mathrm{d} x}{\sqrt{\left(1-v_{L}^{2}\right)\left(1-\phi^{\prime}\right)}}
\end{aligned}
$$

Here $\phi^{\prime}$ is to be expressed as the function of the new coordinates $(t, x)$, i.e. $\phi^{\prime}=\gamma^{-2} \phi$ where $\phi=2 m_{L} /|\Delta x|$, $\Delta x=x_{L}-x$, and $x_{L}=v_{L} t$ is the instantaneous position of the singularity.

The $x_{T}(t)$ null-geodesics describing the world line of $T$ can be obtained by setting $\mathrm{d} s^{2}=0$. Equation A2 shows that there are two solutions

$$
\begin{aligned}
& {\left[v_{L}+\left(1-\gamma^{-2} \phi\right)\right] \mathrm{d} t-\left[1+v_{L}\left(1-\gamma^{-2} \phi\right)\right] \mathrm{d} x_{T}=0} \\
& {\left[v_{L}-\left(1-\gamma^{-2} \phi\right)\right] \mathrm{d} t-\left[1-v_{L}\left(1-\gamma^{-2} \phi\right)\right] \mathrm{d} x_{T}=0}
\end{aligned}
$$

These differential equations can also be obtained more simply by finding the null-geodesics in the comoving coordinates $\left(t^{\prime}, x^{\prime}\right)$ first, and changing to the $(t, x)$ coordinates only in the resulting equation. The null geodesics in the comoving coordinates are simply $\mathrm{d} x_{T}^{\prime} / \mathrm{d} t^{\prime}= \pm\left|1-\phi^{\prime}\right|$ (see Eq. 20), and the Lorentz boost coordinate transformation of this differential equation leads instantly to (A3 A4). Therefore, the two solutions (A3 A4 describe the null geodesics approaching or receding the moving $\mathrm{BH}$, respectively.

We would like to find the solution for the $T$ test particle approaching the source $L$ from behind, namely Eq. (A3) for an initial condition $x_{T}<x_{L}$. This first-order differential equation can be solved analytically by a linear substitution. The coordinate velocity $v_{T}=\mathrm{d} x_{T}(t) / \mathrm{d} t$ monotonously decreases from 1 to $v_{L}$ as the event horizon at $x_{L \text { hor }}(t)=v_{L} t-2 \gamma^{-2} m_{L}$ is approached. In particular if $v_{L}=1$, i.e. the source $L$ has the speed of light in the free-space background, then the trailing test particle $T$ will not be delayed at all, $v_{T}(t)=1$ for all $t$. However, if $v_{L} \ll 1$ then $T$ is considerably affected by the Shapiro delay near the horizon of $L$.

In concluding the description of this model, let us summarize how the clumpy case compares to the spherically symmetric case of expanding radiation shells. First recall that in the spherically symmetric case, $L$ has no effect on $T$ throughout the dynamics regardless of $v_{L}$ or $\Delta r$. In the clumpy case, the gravity of $L$ delays the motion of $T$. The magnitude of this delay is significant only if both of two conditions are violated: (a) $v_{L} \approx 1$ and (b) $\Delta x=x_{L}-x_{T} \gg 2 \gamma^{-2} m_{L}$. What are the "typical numbers" for these quantities? Eq. (20) implies that $v_{L}=1-2 M_{0} / x$ (which is also true in the clumpy case, see $\S$ A 3 , implying that $\gamma^{-2} \sim 4 M_{0} / r$, and for binary mergers $\Delta x \sim 12 \pi M_{0}, m_{L}<M_{0}-M_{f} \lesssim 0.06 M_{0}$, we find that the two cases are equivalent to (a) $x \gg 2 M_{0}$ and (b) $x \gg 0.2 \Delta m \gtrsim 0.01 M_{0}$. Quite clearly, these conditions will not be violated for distances outside the dynamical regime of strong gravity e.g. $x \gtrsim R_{0}=30 M_{0}$. Thus, we expect only very minor modifications relative to the spherically symmetric case, even in the most clumpy case. For a quantitative estimate we need to integrate these modifications over the relevant distances which we describe next.

\section{No remnant, slowly changing $v_{L}(t)$}

Next we consider a slowly changing source velocity $v_{L}(t)$ for the $\mathrm{BH} L$, continue to neglect a remnant $R$, and calculate the motion of $T$ in this spacetime. Since $L$ is assumed to move on a light-like world-line, it is not effected by $R$ and only responds to the background created prior to the production of the bursts. Thus we assume $L$ moves on the null-geodesic of the background as described by (20) with $v_{L}=1-2 m_{T} / x_{L}$.

If $v_{L}$ is slowly changing, we can consider $v_{L}$ to be constant during short time intervals with infinitesimal jumps on their boundaries. We can then find the corresponding world line segments of $T$ by solving the differential equation $\mathrm{A2}$ and matching the boundary conditions of the successive segments by requiring continuity. In the limit that the length of the constant time intervals approaches zero, the world line $x_{T}(t)$ at every instant is given by Eq. A2 with $v_{L}$ now denoting the instantaneous velocity, and $\phi^{\prime}$ referring to the instantaneous value of the potential: $\phi^{\prime}=\gamma^{-2} \phi$, where $\phi=2 m_{L} /|\Delta x|$ with $\Delta x=x_{L}-x$ and $x_{L}=\int v_{L}(t) \mathrm{d} t$. Thus,

$$
\frac{\mathrm{d} x_{T}}{\mathrm{~d} t}=\frac{v_{L}+1-\gamma^{-2} \phi}{1+v_{L}\left(1-\gamma^{-2} \phi\right)}=1-\frac{2 m_{L}\left(1-v_{L}\right)^{2}}{\Delta x-2 m_{L} v_{L}\left(1-v_{L}\right)} .
$$

Substituting $v_{L}=1-2 m_{T} / x_{L}$, the distance between the clumps of radiation satisfies

$$
\frac{\mathrm{d} \Delta x}{\mathrm{~d} t}=\frac{2 m_{T}}{x_{L}}\left(-1+\frac{4 m_{L} m_{T}}{x_{L} \Delta x-4 m_{L} m_{T}\left(1-2 m_{T} x_{L}^{-1}\right)}\right) .
$$

Note that $x_{L}(t)$ can be used to express the width of the packet $\Delta x$ as a function of $x_{L}$. To simplify the result, we use $q_{L}=m_{L} / m_{T}$, set the units to the Schwarzschild radius $2 m_{T}=1$, and express (A6) in terms of the logarithmic distance variable, $y=\ln \left(x_{L}-1\right)$. Then

$$
\frac{\mathrm{d} \Delta x}{\mathrm{~d} y}=-1+\frac{q_{L}}{x_{L} \Delta x-q_{L}\left(1-x_{L}^{-1}\right)}
$$

which to first order in $1 / x_{L}$ becomes

$$
\frac{\mathrm{d} \Delta x}{\mathrm{~d} y}=-1+\frac{q_{L}}{x_{L} \Delta x} .
$$

Equation (A8) shows that to leading order, the wavepacket packet contracts linearly in terms of the logarithmic distance variable $x$. In the spherically-symmetric case, the inner shell $T$ is not influenced by $L$ and so 
$\mathrm{d} x_{T} / \mathrm{d} t=1$ instead of Eq. (A5), leading to $\mathrm{d} \Delta x / \mathrm{d} y=$ -1 . Therefore the distortion of wave-packets in the maximally clumpy case is the same as in the spherically symmetric case to leading order. The difference arises in the next order given by the second term in (A8) describing how the gravity of $L$ Shapiro-delays the motion of $T$. This is typically of order $(4 \times 6 \%) /(12 \pi) \times x_{L}^{-1} \sim 10^{-4}$ for $x_{L} \sim 30 M_{0}$ and gets exponentially smaller for exponentially larger distances.

\section{Remnant included, changing $v_{L}$}

The previous model assumed no remnant (i.e. $m_{R}=$ 0 ), and postulated that $T$ propagated in the spacetime of $L$ by assuming that the spacetime at $T$ was the spacetime of $L$ at the same instant. Here we consider a nonzero $m_{R}$ and account for the retardation of the effect of $L$ as percieved by $T$. We assume a slowly changing velocity and that the gravitational perturbations are sufficiently small to allow simple superposition to leading order. We follow a simplified approach with the following essential assumptions:

1. The initial condition is a spherically symmetric Schwarzschild spacetime centered at $x=0$.

2. $L$ moves on a null-geodesic $x_{L}(t)$ of the initial background metric of $R$ and $T$, i.e. in a Schwarzschild metric centered at $x=0$ for all $t$ and for a mass $m_{R}+m_{T}$. The world-line of $L$ follows (20) accordingly.

3. $T$ moves on a null-geodesic $x_{T}(t)$ of the background metric of $R$ and $L$, which we assume to be a simple superposition $g_{i j}=\eta_{i j}+\delta g_{i j}^{R}+\delta g_{i j}^{L \text {,ret }}$. Here $\delta g_{i j}^{N}=g_{i j}^{N}-\eta_{i j}$ for a given metric, $g_{i j}^{N}$, where $\eta_{i j}$ is the Minkowski metric, $g_{i j}^{R}$ is the metric of the remnant i.e. the Schwarzschild metric centered at $x=0$ for all $t$ with mass $m_{R}$. The metric $g_{i j}^{L}$ is the stationary boosted Schwarzschild metric A1 A2 with mass $m_{L}$, velocity $v_{L}$, centered at $x_{L}$. The label ${ }^{\text {ret }}$ stands for retardation, which we describe next separately.

4. We account for retardation by setting $\Delta t_{\text {ret }}=t-$ $t_{\text {ret }}$ to be the light-travel time from $L$ to $T$. For this we compute the inward propagating null-geodesics from $L$ to $T$ [i.e. between positions $\left(t_{\text {ret }}, r_{L}\left(t_{\text {ret }}\right)\right)$ and $\left.\left(t, r_{T}(t)\right)\right]$, based on the initial background metric of $R$ and $T$ (i.e. neglecting the gravity of $L$ ).

To find the retardation time, we note that the inward propagating null geodesics satisfies $\mathrm{d} x / \mathrm{d} t=-\left[1-2\left(m_{R}+\right.\right.$ $\left.\left.m_{T}\right) / x\right]$. Since this is exactly the time-reversed world line of $L$, we get $x_{T}(t)=x_{L}\left(t-2 \Delta t_{\text {ret }}\right)$. Integrating $\mathrm{d} t / \mathrm{d} x$ for the world line of $L$ between $x_{L}\left(t-2 \Delta t_{\text {ret }}\right)$ and $x_{L}(t)$,

$$
2 \Delta t_{\mathrm{ret}}=\int_{x_{T}(t)}^{x_{L}(t)} \frac{x_{L}}{x_{L}-2 m_{R}-2 m_{T}} \mathrm{~d} x_{L},
$$

from which

$$
\Delta t_{\mathrm{ret}}=\frac{x_{L}-x_{T}}{2}+\left(m_{R}+m_{T}\right) \ln \frac{x_{L}-2 m_{R}-2 m_{T}}{x_{T}-2 m_{R}-2 m_{T}} .
$$

The distance where $T$ percieves $L$ is $x_{L}^{\mathrm{ret}}(t)=x_{L}(t-$ $\left.\Delta t_{\text {ret }}\right)$ and the separation is $\Delta x_{\text {ret }} \equiv x_{L}^{\text {ret }}-x_{T}$. Substituting $x_{T}$ as $x_{T}=x_{L}-\Delta x$ and $x_{T}=x_{L}^{\text {ret }}-\Delta x_{\text {ret }}$ into (A10), we can find $\Delta x_{\text {ret }}$ for given $\Delta x$ and $x_{L}$. To first nonvanishing order in $1 / x_{L}$,

$$
\Delta x_{\mathrm{ret}}=\frac{\Delta x}{2}-\frac{\left(m_{R}+m_{T}\right) \Delta x^{2}}{4 x_{L}^{2}} \text {. }
$$

The leading order term corresponds to the propagation at the speed of light in free space, $v_{L}=v_{T}=1$. Note that the correction is proportional to $x_{L}^{-2}$, which is extremely small for the physical cases beyond the strong field zone. Finally, we define the retarded position and velocity $x_{L}^{\text {ret }}=x_{L}-\Delta x_{\text {ret }}$, and $v_{L}^{\text {ret }}=1-2\left(m_{R}+m_{T}\right) / x_{L}^{\text {ret }}$, which can be written in terms of $x_{L}$ and $\Delta x$ using Eq. A11.

The metric contribution $\delta g_{i j}^{L \text {,ret }}$ of $L$ at $T$ at time $t$, is the boosted Schwarzschild metric A1A2 with instantaneous velocity $v_{L}^{\text {ret }}$, a singularity at $x_{L}^{\text {ret }}$, and distance $\Delta x_{\text {ret }}$.

Now we can redo the derivation presented in $\S \mathrm{A} 2$ to find the motion of $T$, using the modified spacetime $g_{i j}$ given above. Again we find two solutions for $\mathrm{d} s^{2}=0$ representing the ingoing and outgoing radiation. Expanding the outgoing solution in a series in $x_{L}^{-1}$, we find

$$
\left.\frac{\mathrm{d} x_{T}}{\mathrm{~d} t}\right|_{\text {clumpy }}=1-\frac{2 q_{R}}{x_{L}}-\left[q_{R} \Delta x+\frac{2 q_{L}\left(1+q_{R}^{2}\right)}{\Delta x}\right] \frac{1}{x_{L}^{2}},
$$

where $q_{i}=m_{i} /\left(m_{R}+m_{T}\right)$ for $i \in\{R, T, L\}$ and distance units are chosen to be the Schwarzschild radius $2\left(m_{R}+\right.$ $\left.m_{T}\right) \equiv 1$. In order to get the instantaneous shell width $\Delta x$ as a function of logarithmic distance $y$, we can redo the manipulations of (A6 A8 for the result (A12). To first order in $1 / x_{L}$,

$$
\left.\frac{\mathrm{d} \Delta x}{\mathrm{~d} y}\right|_{\text {clumpy }}=-q_{T}+\left[q_{R} \Delta x+\frac{2 q_{L}\left(1+q_{R}^{2}\right)}{\Delta x}\right] \frac{1}{x_{L}} .
$$

In the limit of no remnant $q_{R}=0$, we almost recover the solution derived previously in Eq. (A8). There is a factor 2 difference, which is the direct consequence of the retardation of the percieved distance $\Delta x$, which had been neglected in Eq. (A8).

Equation A13 should be contrasted to the expansion of two spherically symmetric shells $m_{T}$ and $m_{L}$ in the presence of a remnant $m_{R}$. We may expand the corresponding spherical solution of $\S$ IIIC in a series in $1 / x_{L}$ to first order:

$$
\left.\frac{\mathrm{d} \Delta x}{\mathrm{~d} y}\right|_{\text {spherical }}=-q_{T}+\frac{q_{R} \Delta x}{x_{L}} .
$$


The first two terms in Eqs. (A13) and (A14) are identical. The correction describing the "Shapiro delay" of contraction in the clumpy case due to the gravity of $L$ is $2 q_{L}\left(1+q_{R}^{2}\right) /\left(x_{L} \Delta x\right)$. Substituting typical physical values $q_{R}=97 \%, q_{T}=3 \%, q_{R}=3 \%$, and $\Delta x_{0} \sim 6 \pi \sim x_{L 0}$ in units of Schwarzschild radii, we see that the correc- tion is of order $10^{-4}$ initially, and becomes exponentially smaller at exponentially larger distances. In summary, even in the most extreme case of clumpiness, the radiation packet propagates to very high precision according to the spherically symmetric description.
[1] F. Pretorius, Phys. Rev. Lett. 95, 121101 (2005), arXiv:gr-qc/0507014.

[2] M. Campanelli, C. O. Lousto, P. Marronetti, and Y. Zlochower, Phys. Rev. Lett. 96, 111101 (2006), arXiv:grqc/0511048.

[3] J. G. Baker, J. Centrella, D.-I. Choi, M. Koppitz, and J. van Meter, Phys. Rev. Lett. 96, 111102 (2006), arXiv:gr-qc/0511103.

[4] J. G. Baker, S. T. McWilliams, J. R. van Meter, J. Centrella, D.-I. Choi, B. J. Kelly, and M. Koppitz, ArXiv General Relativity and Quantum Cosmology e-prints (2006), gr-qc/0612117.

[5] E. Pazos, E. N. Dorband, A. Nagar, C. Palenzuela, E. Schnetter, and M. Tiglio, Classical and Quantum Gravity 24, 341 (2007), arXiv:gr-qc/0612149.

[6] I. I. Shapiro, Phys. Rev. Lett. 13, 789 (1964).

[7] P. C. Vaidya, Physical Review 83, 10 (1951).

[8] P. C. Vaidya and K. B. Shah, Progress of Theoretical Physics 24, 111 (1960).

[9] D. R. Brill and J. B. Hartle, Physical Review 135, 271 (1964).

[10] R. A. Isaacson, Physical Review 166, 1263 (1968).

[11] R. A. Isaacson, Physical Review 166, 1272 (1968).

[12] C. W. Misner, K. S. Thorne, and J. A. Wheeler, Gravitation (San Francisco: W.H. Freeman and Co., 1973, 1973).

[13] K. S. Thorne and S. J. Kovacs, ApJ 200, 245 (1975).

[14] K. S. Thorne, Reviews of Modern Physics 52, 299 (1980).

[15] M. Efroimsky, Phys. Rev. D 49, 6512 (1994).

[16] T. Regge and J. A. Wheeler, Physical Review 108, 1063 (1957).

[17] F. J. Zerilli, Phys. Rev. D 2, 2141 (1970).

[18] V. Moncrief, Annals of Physics 88, 323 (1974).

[19] B. Brügmann, J. A. González, M. Hannam, S. Husa, U. Sperhake, and W. Tichy, ArXiv General Relativity and Quantum Cosmology e-prints (2006), gr-qc/0610128.

[20] J. Baker, M. Campanelli, C. O. Lousto, and R. Takahashi, Phys. Rev. D 69, 027505 (2004), arXiv:astro$\mathrm{ph} / 0305287$.

[21] M. Campanelli, C. O. Lousto, and Y. Zlochower, Phys. Rev. D 73, 061501(R) (2006), arXiv:grqc/0601091.

[22] M. Campanelli, C. O. Lousto, and Y. Zlochower, Phys. Rev. D 74, 041501(R) (2006), arXiv:grqc/0604012.

[23] W. Tichy and P. Marronetti, ArXiv General Relativity and Quantum Cosmology e-prints (2007), gr-qc/0703075.

[24] F. Pretorius and D. Khurana, Classical and Quantum Gravity 24, 83 (2007), arXiv:gr-qc/0702084.

[25] E. Berti, V. Cardoso, J. A. Gonzalez, U. Sperhake, M. Hannam, S. Husa, and B. Bruegmann, ArXiv General Relativity and Quantum Cosmology e-prints (2007), gr-qc/0703053.
[26] A. Buonanno, G. B. Cook, and F. Pretorius, Phys. Rev. D 75, 124018 (2007), arXiv:gr-qc/0610122.

[27] J. G. Baker, J. Centrella, D.-I. Choi, M. Koppitz, and J. van Meter, Phys. Rev. D 73, 104002 (2006), arXiv:grqc/0602026.

[28] L. Blanchet and T. Damour, Physics Letters A 104, 82 (1984).

[29] L. Blanchet and T. Damour, Royal Society of London Philosophical Transactions Series A 320, 379 (1986).

[30] L. Blanchet and T. Damour, Phys. Rev. D 46, 4304 (1992).

[31] L. Blanchet, Living Reviews in Relativity 9, 4 (2006).

[32] K. G. Arun, Phys. Rev. D 74, 024025 (2006), arXiv:grqc/0605021.

[33] T. Tanaka, H. Tagoshi, and M. Sasaki, Progress of Theoretical Physics 96, 1087 (1996), arXiv:gr-qc/9701050.

[34] L. Blanchet, Classical and Quantum Gravity 15, 1971 (1998), arXiv:gr-qc/9801101.

[35] A. G. Wiseman and C. M. Will, Phys. Rev. D 44, R2945 (1991).

[36] E. Poisson, Phys. Rev. D 47, 1497 (1993).

[37] A. G. Wiseman, Phys. Rev. D 48, 4757 (1993).

[38] L. Blanchet and G. Schäfer, Classical and Quantum Gravity 10, 2699 (1993).

[39] L. Blanchet, Classical and Quantum Gravity 15, 89 (1998), arXiv:gr-qc/9710037.

[40] L. Blanchet, Classical and Quantum Gravity 15, 113 (1998), arXiv:gr-qc/9710038.

[41] D. Christodoulou, Phys. Rev. Lett. 67, 1486 (1991).

[42] K. S. Thorne, Phys. Rev. D 45, 520 (1992).

[43] R. J. Gleiser and A. E. Domínguez, Phys. Rev. D 68, 104018 (2003), arXiv:gr-qc/0306085.

[44] W. B. Bonnor, Communications in Mathematical Physics 13, 163 (1969).

[45] P. C. Aichelburg and R. U. Sexl, Gen. Rel. Grav. 2, 303 (1971).

[46] K. Hayashi and T. Samura, Phys. Rev. D 50, 3666 (1994), arXiv:gr-qc/9404027.

[47] J. T. Mendonça and V. Cardoso, Phys. Rev. D 66, 104009 (2002), arXiv:gr-qc/0209076.

[48] M. Servin, M. Marklund, G. Brodin, J. T. Mendonça, and V. Cardoso, Phys. Rev. D 67, 087501 (2003), arXiv:astro$\mathrm{ph} / 0303412$.

[49] J. T. Mendonça, V. Cardoso, M. Marklund, M. Servin, and G. Brodin, Phys. Rev. D 68, 084025 (2003), arXiv:gr-qc/0307031.

[50] E. Nakar, A. Gal-Yam, and D. B. Fox, ApJ 650, 281 (2006), arXiv:astro-ph/0511254.

[51] R. M. O'Leary, F. A. Rasio, J. M. Fregeau, N. Ivanova, and R. O'Shaughnessy, ApJ 637, 937 (2006), arXiv:astro-ph/0508224.

[52] S. F. Portegies Zwart and S. L. W. McMillan, ApJ 528, L17 (2000), arXiv:astro-ph/9910061. 
[53] J. S. B. Wyithe and A. Loeb, ApJ 610, 117 (2004), arXiv:astro-ph/0401554.

[54] S. F. Portegies Zwart, H. Baumgardt, S. L. W. McMillan, J. Makino, P. Hut, and T. Ebisuzaki, ApJ 641, 319 (2006), arXiv:astro-ph/0511397.

[55] M. Micic, K. Holley-Bockelmann, S. Sigurdsson, and T. Abel, ArXiv Astrophysics e-prints (2007), astro$\mathrm{ph} / 0703540$.

[56] J. S. B. Wyithe and A. Loeb, ApJ 590, 691 (2003), arXiv:astro-ph/0211556.

[57] A. Sesana, F. Haardt, P. Madau, and M. Volonteri, Classical and Quantum Gravity 22, 363 (2005), arXiv:astro$\mathrm{ph} / 0502462$.

[58] B. Kocsis, M. E. Gáspár, and S. Márka, ApJ 648, 411 (2006), arXiv:astro-ph/0603441.

[59] J. G. Baker, M. Campanelli, F. Pretorius, and Y. Zlochower, Classical and Quantum Gravity 24, 25 (2007), arXiv:gr-qc/0701016.

[60] F. Löffler, L. Rezzolla, and M. Ansorg, Phys. Rev. D 74, 104018 (2006), arXiv:gr-qc/0606104.

[61] M. Campanelli, C. O. Lousto, and Y. Zlochower, Phys. Rev. D 74, 084023 (2006), arXiv:astro$\mathrm{ph} / 0608275$.

[62] W. H. Lee and W. Ł. Kluźniak, MNRAS 308, 780 (1999), arXiv:astro-ph/9904328.

[63] W. H. Lee and W. Ł. Kluźniak, ApJ 526, 178 (1999), arXiv:astro-ph/9808185.

[64] U. Sperhake, B. Kelly, P. Laguna, K. L. Smith, and E. Schnetter, Phys. Rev. D 71, 124042 (2005), arXiv:grqc/0503071.

[65] M. Alcubierre, W. Benger, B. Brügmann, G. Lanfermann, L. Nerger, E. Seidel, and R. Takahashi, Phys. Rev. Lett. 87, 271103 (2001), arXiv:gr-qc/0012079.

[66] P. D. D'Eath and P. N. Payne, Phys. Rev. D 46, 694
(1992).

[67] V. Cardoso and J. P. S. Lemos, Phys. Rev. D 67, 084005 (2003), arXiv:gr-qc/0211094.

[68] R. M. Wald, General relativity (Chicago, University of Chicago Press, 1984).

[69] V. A. Fock, Theory of space time and gravitation (New York, Pergamon Press, 1959).

[70] R. A. Isaacson and J. Winicour, Physical Review 168, 1451 (1968).

[71] J. Madore, Annales de l'institut Henri Poincaré (A) Physique théorique 12, 285 (1970).

[72] L. Lehner and O. M. Moreschi, ArXiv e-prints 706 (2007), 0706.1319.

[73] R. N. Lang and S. A. Hughes, Phys. Rev. D 74, 122001 (2006), arXiv:gr-qc/0608062.

[74] R. N. Lang and S. A. Hughes, Phys. Rev. D 75, 089902(E) (2007).

[75] D. E. Holz and S. A. Hughes, ApJ 629, 15 (2005), arXiv:astro-ph/0504616.

[76] http://www.ligo.caltech.edu/

[77] http://www.virgo.infn.it/

[78] http://tamago.mtk.nao.ac.jp/

[79] http://geo600.aei.mpg.de/

[80] http://www.ligo.caltech.edu/advLIGO/

[81] http://wwwcascina.virgo.infn.it/advirgo/

[82] http://www.icrr.u-tokyo.ac.jp/gr/LCGT.html

[83] http://www.lisa-science.org/

[84] Throughout this paper we assume that the metric at $r \rightarrow \infty$ is the Minkowski metric, neglecting cosmological effects. This is justified by the orders of magnitudes or radii where the radiation shell distortion is relevant $r<10^{3} M_{0}$ (see Fig. 2) is much less than cosmological scales $r \gtrsim 10^{18} M_{0}$. 